\title{
Effectiveness of health systems strengthening interventions in Africa: a systematic review
}

\author{
Olaiya O. Fatodu*
}

c/o Malaysia Institute for Supply Chain Innovation, No. 2A, Persiaran Tebar Layar, Seksyen U8,

Bukit Jelutong, Shah Alam, 40150 Selangor, Malaysia

Email: olapharm@gmail.com

*Corresponding author

\section{Olusola O. Oyenuga}

Rite Aid Pharmacy,

Edgewood, MD, USA

Email: solapharm@gmail.com

\begin{abstract}
World Health Organization devoted World Health Report of 2000 to health systems performance. Since then, many international development partners and national governments have been funding health systems strengthening (HSS) interventions. Interventions that are included in this review are those that were carried out in single or multiple African countries. Among other criteria, such interventions had to directly target at least one of six HSs building blocks. Eighteen relevant evaluations were critically appraised for quality from 200 potentially relevant evaluations. No intervention concurrently strengthened all the six HSs building blocks and none improved all four overall outcomes of HSS. HSS intervention in Africa deserves more attention by policy makers, global health funders, and public health professionals in Africa. This systematic review reveals shortcomings of HSS interventions in Africa and makes four evidence-based recommendations to guide and improve future HSS interventions in Africa to enable them have impacts on all intermediate and overall HSS outcomes indicators.
\end{abstract}

Keywords: health systems strengthening; HSS; health systems; Africa; health systems intervention.

Reference to this paper should be made as follows: Fatodu, O.O. and Oyenuga, O.O. (2019) 'Effectiveness of health systems strengthening interventions in Africa: a systematic review', Int. J. Healthcare Policy, Vol. 1, No. 1, pp.52-69.

Biographical notes: Olaiya $O$. Fatodu is a multi-skilled pharmacist with multiple Master degrees. He earned his MBA as University Scholar at the University of Exeter Business School, UK, and completed his MPH in Global Health as UK Commonwealth Scholar at the University of Manchester, UK. He earned his MSc in Supply Chain Management from the Malaysia Institute for Supply Chain Innovation (MIT Global SCALE Network member in Asia). His practice and research interests are health systems development, performance improvement in service delivery, service business growth, leadership development, capacity building in people management, and supply chain management in both public and private sectors. 
Olusola O. Oyenuga is a multi-countries registered pharmacist. He earned his BPharm from the University of Lagos, Nigeria and Postgraduate Diploma in HIV/AIDS Management from the University of Stellenbosch, South Africa. He currently practices pharmacy in Maryland, USA.

\section{Introduction}

Over a century ago, in advanced countries, structured health systems (HSs) that catered to health needs of the populations (as defined in contemporary times) did not exist (WHO, 2000). To stress importance of HSs, in contemporary times, Sekhri (2006), writes that performance of HSs could decide who lives and who dies. This emphasises the importance that must be attached to HSs. World Bank (2004) buttresses this position by stating that a young woman's risk of death in childbirth in Uganda is 300 times higher than that of a young woman in the USA. Similarly, a child born in Niger compared to another child born in the UK, is 40 times less likely to celebrate his/her fifth birthday, and a boy born in Swaziland has 18\% chance of celebrating his 60th birthday, whereas the same boy, if born in Switzerland, would have 91\% chance (WHO, 2006). In above cited cases, in addition to other factors, performance of HSs makes huge difference in life expectancy of individuals and population. It was recognition of these crucial contributions of functioning HSs to society that World Health Organisation (WHO) devoted World Health Report of 2000 to HSs performance.

HSs have been defined by Tanahashi (1978), Sekhri (2006), and WHO (2007, 2008). HSs are composite and context-sensitive (WHO, 2007, 2010b), therefore, there are no universal practices that could be recommended to improve HSs in different contexts. Sekhri (2006) writes that weak HSs are one of the chronic challenges which have limited major health gains and economic development in Africa, as millions of people find it difficult to access basic health services because they are not affordable and/or such health services are non-existent in their communities. WHO $(2000,2007)$ presented a framework with six building blocks that constitute HS and modified by these authors as shown in Figure 1.

Health systems strengthening (HSS) in its broadest definition is any set of interventions and strategies that improves at least one of the functions of a HS, and that results in better health through an increase in access, coverage, quality, or efficiency (Islam, 2007). Other definitions are by WHO (2007, 2010b).

In agreement with position of Richards (2004), this systematic review (SR) is focused on topic of importance to seemingly resource-poor countries, African countries. Emphasising this need, Sekhri (2006), writes that of all 191 countries surveyed in World Health Report 2000 (WHO, 2000), most of the nations in sub-Sahara Africa were categorised in the bottom $50 \%$ based on performance of their HSs. Knowing that HSs performance could make a difference between life and death, and sequel to World Health Report 2000, big funders of global health, such as The Global Fund to Fight AIDS, Tuberculosis and Malaria (Global Fund), the GAVI Alliance (GAVI), and the President's Emergency Plan for AIDS Relief (PEPFAR), for example, have directed funds towards HSS (WHO, 2000, 2007; Shakarishvili et al., 2011; Hafner and Shiffman, 2013). All 
these justify the focus of this study on HSS interventions that took place in Africa between 2000 and 2013.

Figure 1 Modified WHO HS framework

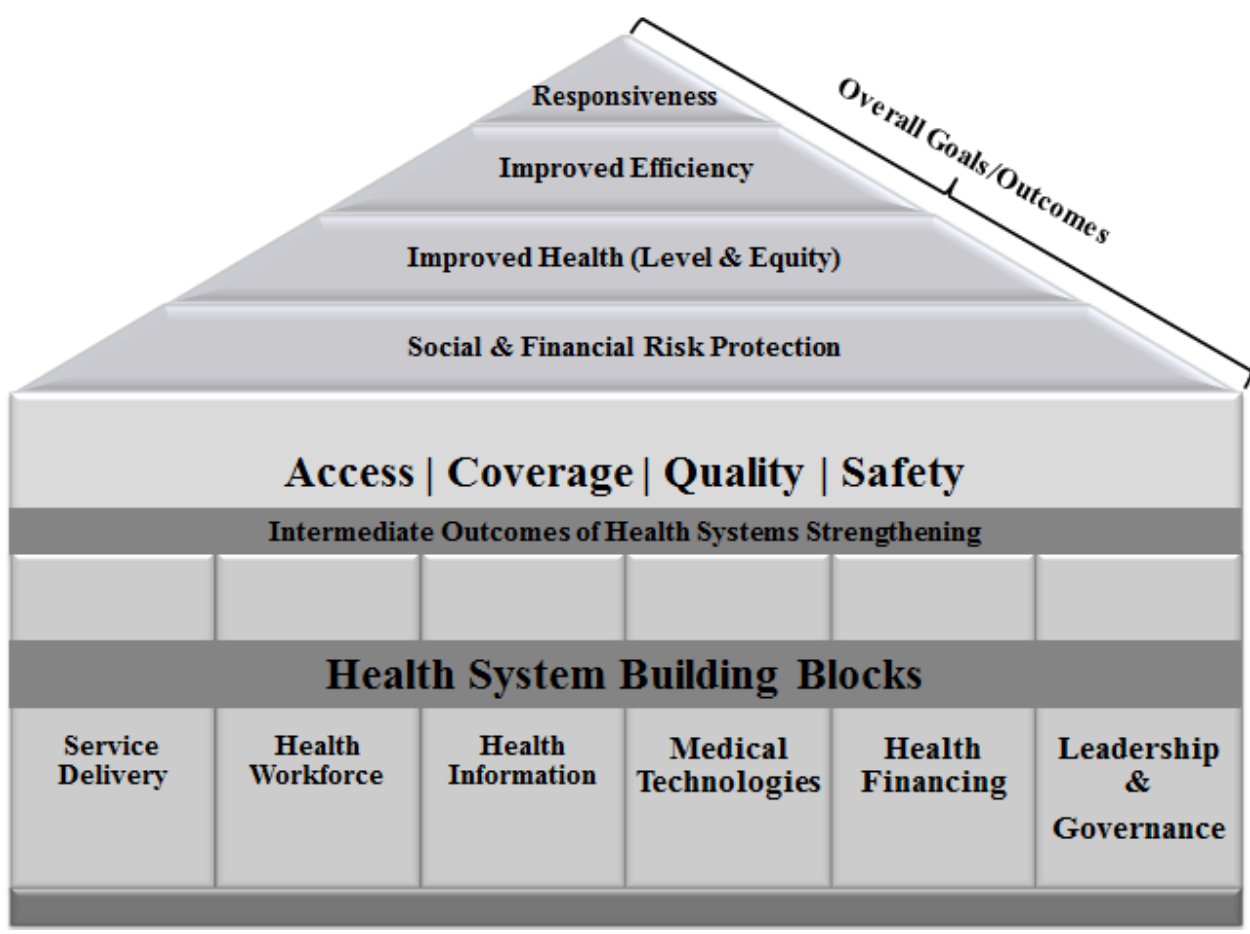

Source: Adapted from WHO (2007)

\subsection{Previous SRs on effectiveness of HSS interventions}

Literature on impacts of HSS interventions in low and middle income countries (LMICs) is limited. This reflects the view of Adam et al. (2012) who report that impacts of HSS interventions particularly in LMICs have not been extensively researched. They write in their review of peer-reviewed and grey literature prior 2008 that they found limited number of evaluations assessing broader impact of interventions on HSs. Their work is focused on whether intervention studies asked the type of question(s) that capture the system-wide effects of such intervention. Searching literature, just one SR paper was found on impact of HSS interventions in Africa or any African countries. The paper is a review of HSS interventions in Rwanda by Bucagu et al. (2012). In it, Bucagu et al. (2012) argue that in Rwanda, HSS intervention directed at three building blocks of HSs (human resources, financing, and governance) resulted in improvement of secondary outcomes - infant mortality, and under-5-child mortality as listed by Cohen et al. (2013).

Furthermore, there is dearth of research on impacts of HSS interventions across multiple countries in Africa. To the limited knowledge of authors of this paper, this SR will arguably be the first SR of HSS interventions that will cut across multiple African countries. Most importantly, this SR is set to find out the extent to which HSS 
interventions in Africa resulted in improved HSs as measured by indicators of HSs building blocks and distal indicators of HSS, and also seeks to establish if HSS interventions are indeed yielding the expected result(s). For the purpose of this study, HSS interventions are defined as interventions that directly targeted at least one of the six HSs building blocks and their sub-components as defined by WHO (2007); or disease-specific interventions or programs that also have important system-wide impacts, e.g., scale-up of antiretroviral therapy for HIV/AIDS (de Savigny and Adam, 2009; Adam et al., 2012).

\section{Methods}

\subsection{Literature search and screening}

Medline and Embase were concurrently and systematically searched for articles via Ovid. The search was also conducted on individual websites of big funders of global health (Global Fund, GAVI, and PEPFAR) who are known to be funding HSS interventions. Hand searching was not considered for this review due to location and other constraints. Qualitative and quantitative evaluations (non-peer reviewed articles) that met the inclusion criteria were retrieved and included. The inclusion criteria are:

- Evaluations of HSS interventions were undertaken in one African country or undertaken simultaneously in multiple African countries.

- Interventions that directly targeted at least one of six HSs building blocks and their subcomponents as defined by the WHO (2007); or disease-specific interventions or programs that also have important system-wide impacts, e.g., scale-up of antiretroviral therapy for HIV/AIDS (de Savigny and Adam, 2009; Adam et al., 2012).

- Intervention must have impacted at least, intermediate goals of HSS (increased access; increased coverage; ensuring provider quality and safety), and/or overall goals/outcomes of HSS (responsiveness; improved efficiency; improved health level and equity; social and financial risk protection).

- Published and unpublished evaluations of HSS interventions that were carried out between 2000 and 2013, and are in English language.

The following search terms were used: HSS; health system strengthening; health-system strengthening; health-systems strengthening; strengthening health systems; and strengthening health system; Africa*.

Two authors (OF and OO) separately screened the retrieved peer-reviewed papers as well as grey literatures for relevance based on the inclusion criteria. Once both authors agreed on relevance of a paper, such paper was included. Border line papers and grey literatures were consensually screened in or out. 200 (150 from Medline and Embase, 49 from grey literatures, and one from evaluation references) potentially relevant evaluations were gotten. This number was reduced to 18 very relevant evaluations (seven peer-reviewed articles and 11 reports of intervention evaluations) that satisfied all inclusion criteria. Exclusions were for various reasons: 55 articles were removed electronically and three articles were removed manually for duplication reason; 
119 seemingly relevant articles/reports, but not fully relevant were also removed. One study in French language with abstract in English language was eliminated; and four articles/journals were excluded after reassessment and disagreement resolution by both authors.

\subsection{Data extraction}

Data extraction was carried out by both authors of this SR. The extraction template is an adapted version of Adam et al. (2012). The following variables were considered and included for data extraction:

1 author(s) of included evaluations

2 country or countries where evaluation was undertaken

3 type of intervention (system-level or disease-specific)

4 HSs building block(s) targeted by interventions

5 type of impacts assessed (intermediate goals and/or overall outcomes of HSS)

6 results and specific HSS outcomes improved.

See Appendix 1 for extracted data.

\subsection{Assessment of study quality}

Two tools, quality appraisal checklist - qualitative studies, and quality appraisal checklist - quantitative intervention studies by NICE (2012) were used in critical assessment of included evaluations for quality, since the review included both qualitative and quantitative evaluations as suggested by WHO (2010a). Summaries of critical quality assessments of included qualitative and quantitative evaluations are shown in Appendices 2 and 3, respectively.

\subsection{Summary of included evaluations}

\subsubsection{Types of settings}

There are total of 18 studies/reports included in this review. Two of the studies covered multiple countries: Duber et al. (2010) and Cohen et al. (2013). The rest covered 13 individual African countries. Two studies covered South Africa: Youngleson et al. (2010) and Webster et al. (2012). Rwanda and Ethiopia are each covered by both a report and a study. The remaining ten studies/reports covered ten countries. Ten of the reports were commissioned by GAVI and 1 by United States Agency for International Development (USAID). See Appendix 1 for extracted data details. The two studies that covered multiple countries are retrospective analysis of publicly available health outcome data.

\subsubsection{Types of interventions and impacts}

There are four studies that evaluated disease-specific interventions [Duber et al. (2010) HIV/AIDS/multiple countries; Youngleson et al. (2010) - HIV/AIDS/South Africa; 
Webster et al. (2012) - HIV/AIDS/South Africa; and Cohen et al. (2013) HIV/AIDS/multiple countries]. The remaining 14 studies/reports evaluated system level interventions. Out of the 14 studies/reports that evaluated system level interventions, three are studies while 11 are reports.

\subsubsection{HSs building block(s) targeted by interventions}

Understandably, two multi-countries retrospective data analysis studies did not report on specific HSS building block(s) targeted in interventions they evaluated. All evaluations that reported on HSS building blocks targeted at least one building block for strengthening. No study reported on intervention that targeted all the six building blocks. The breakdown is shown in Table 1.

Table 1 HSs building blocks and number of studies/reports

\begin{tabular}{lc}
\hline Health systems building block & Number of studies/reports \\
\hline Health workforce & 15 \\
Health services delivery & 12 \\
Health financing & 2 \\
Leadership and governance & 4 \\
Health information & 3 \\
Medical products, technologies and vaccines & 6 \\
\hline
\end{tabular}

\subsubsection{Type of impacts assessed}

HSS interventions have impacts at two levels: intermediate outcomes (access, coverage, quality, and safety) and overall outcomes (improved health, responsiveness, health efficiency, and social and financial risk protection). All the seven peer-reviewed studies included in this SR reported impacts of HSS interventions at both levels. Of the 11 reports included in this SR, seven [Smith et al. (2005) - Benin; Carlson et al. (2009) Democratic Republic of Congo; England (2009) - Ghana; Lewis (2009a) - Nigeria; Martinez and Karasi (2009) - Rwanda; Lewis (2009b) - Sierra Leone; and Lewis and Kamanga (2009) - Zambia] did not report on impacts of interventions for reasons of interventions being in early stage or probably it was a case of omission during report writing.

\section{Results}

The evaluations included in this SR are in two groups: quantitative and qualitative. Among the 12 evaluations in the qualitative group are ten studies tagged 'GAVI HSS support evaluations'. They were commissioned by GAVI, and conducted by HLSP Institute, UK. The remaining evaluation is a study sponsored by USAID to evaluate system-wide effects of Global Fund in Benin. The ten 'GAVI HSS evaluation' reports collectively have weak evidence base to support their findings. The reports cited positive impacts of HSS interventions without providing tangible evidence to support them.

In summary, under intermediate outcomes of HSS, nine evaluations representing $50 \%$ of all evaluations reviewed showed effects on access, and coverage. Six evaluations 
representing 33\%, had impacts on quality, while one study by Kebede et al. (2012), representing $6 \%$ impacted safety. Under overall outcomes of HSS, ten evaluations representing $56 \%$ of all evaluations had effects on improved health. This is followed by improvement in responsiveness reported in nine evaluations representing $50 \%$ of all evaluations. Two evaluations representing 11\% showed effect on improved efficiency, while one study by Kalk et al. (2010), representing 6\% impacted social and financial risk protection.

\subsection{Coverage of overall HSS outcomes}

None of the evaluations of HSS interventions included in this SR shows improvement of all the four overall outcomes of HSS. Improved health and responsiveness are the most commonly improved HSS overall outcomes. Seven evaluations of HSS interventions demonstrated improvement of two overall outcomes of HSS. It is only a study by Pfeiffer et al. (2010) which showed that HSS intervention improved three HSS overall outcomes (improved efficiency, improved health, and responsiveness). It is only a study by Kalk et al. (2010) in Rwanda that improved social and financial risk protection as HSS overall outcome. Seven reports (Smith et al., 2005; Carlson et al., 2009; England, 2009; Lewis, 2009a; 2009b, Martinez and Karasi, 2009; Lewis and Kamanga, 2009) did not include any HSS overall outcome that was impacted. Six of the reports were from studies commissioned by GAVI.

\subsection{Applicability of findings from included evaluations}

There are many similarities within each regional bloc in Africa. This may explain reason for unintentional exclusion of any North African countries in this review. North African countries are either Arabic or French speaking, therefore, their publications are in either of the two languages which did not meet selection criteria for this SR. Due to intra-regional similarities in Southern Africa, findings of Youngleson et al. (2010) and Webster et al. (2012) may apply in other provinces in South Africa and other Southern African countries like Botswana, Namibia, etc. Similarly, findings of Kebede et al. (2012) in Ethiopia may apply in other East African countries wherever hospitals have established posts of Chief Executive Officer who are undergoing or have undergone a two-year executive Master of Health Administration program. Probably findings of Kebede et al. (2012) can also apply in other regions of Africa wherever there are political will and resources to make it work.

Findings of Kalk et al. (2010) in Rwanda will particularly be applicable in East Africa and any other African countries where there are good citizen identification and accountability systems because of money disbursement and management that are involved. Most of the HSS interventions sponsored by major global health funders are directed at HIV/AIDS, malaria and tuberculosis ravaged countries as shown by the 18 evaluations included in this SR. Therefore, it is unclear if the findings from reports of these HSS interventions may be applicable in non-PEPFAR and non-Global Fund countries in Africa where there may be needs for HSS interventions as well. Besides the reports and multi-countries studies, there was no study from any specific West African countries included in this SR. This implies that the findings from studies in Southern Africa and East Africa may not apply in any of the West African countries 


\subsection{Quality of evidence of included evaluations}

Based on NICE (2012) quality appraisal for qualitative studies, five of the 12 qualitative evaluations included in this SR performed poorly with respect to overall quality assessment. Four reports performed fairly well, while three reports fared very well in terms of overall quality assessment. The two authors (also reviewers) were 'not sure' if findings from all the 12 qualitative evaluations were convincing because they did not provide extracts from any original data. Four reports (Carlson and Karibwami, 2009; England, 2009; Alebachew and Ortendahl, 2009b; Lewis, 2009b) were considered to have used 'unreliable' method because they did not triangulate data source neither did they provide justification for this inaction.

Ten reports (Carlson and Karibwami, 2009; Carlson et al., 2009; Alebachew and Ortendahl, 2009a, 2009b; England, 2009; Lewis and Leigh, 2009; Lewis, 2009a, 2009b; Martinez and Karasi, 2009; Lewis and Kamanga, 2009) are considered to have used insufficiently 'rigorous' data analysis because the procedure they used are not explicit, and it is not clear how data led to themes and concepts in the reports. Ten of the reports came up with findings relevant to the aims of the studies while two reports have findings partially relevant to aims of the studies.

Nine of the qualitative evaluations (Kalk et al., 2010; Smith et al., 2005; Alebachew and Ortendahl, 2009a, 2009b; England, 2009; Lewis and Leigh, 2009; Lewis, 2009a, 2009b; Martinez and Karasi, 2009) inadequately discussed any limitations encountered in their research process. The limitations could have influenced outcomes of the evaluations as well as possibly mislead interpretation and application of their evaluations results.

Using NICE (2012) quality appraisal for quantitative studies, five of the six quantitative studies included in this SR provided thorough description of study source population/areas/countries. All the six studies had eligible population/areas/countries that were representative of the source population just as the selected study participants were all representative of eligible population/areas/countries. Studies by Duber et al. (2010) and Cohen et al. (2013) minimised selection bias through criteria used in selecting exposure and comparison groups. Two studies (Pfeiffer et al., 2010; Youngleson et al., 2010) did not report on selection of intervention and comparison groups, while selection bias is not applicable in studies by Kebede et al. (2012) and Webster et al. (2012). All the six studies measured relevant outcomes in a reliable manner. Two studies (Kebede et al., 2012; Webster et al., 2012) are fairly internally valid because they endeavoured to minimise effect of sources bias by warning that their findings should be considered with caution. The remaining four studies are considered internally invalid.

\subsection{Comparison with other SRs}

The authors (OF and OO) of this SR are aware of limited literature that systematically reviewed evaluations of HSS interventions. Therefore, comparison is done with SRs by Adam et al. (2012) and Bucagu et al. (2012).

This SR and that of Adam et al. (2012) share same definition of HSS. Both Adams et al. (2012) and this SR searched Medline and Embase for their peer-reviewed articles. In this SR, 12 out of 18 evaluations focused on system-level HSS interventions just as Adam et al. (2012) found out that most HSS interventions are system-level interventions. Like review by Adam et al. (2012), this SR found out that most evaluations of 
disease-specific-HSS interventions assessed HIV/AIDS scale-up services or effect of disease-specific global health initiatives on HSs.

This SR and Adam et al. (2012) have more dissimilarities than similarities. Focus of this SR is Africa while that of Adam et al. (2012) is wider, LMICs. The authors of this SR assessed quality of HSS interventions evaluations included in this SR while Adam et al. (2012) disregarded critical quality assessment of included studies. Since focus of SR by Adam et al. (2012) is wider than that of this SR, it is understandable that Adam et al. (2012) systematically reviewed 106 HSS interventions evaluations while this study systematically reviewed just 18 HSS intervention evaluations. This SR found out that 15 evaluations representing $83 \%$ of the evaluations assessed impact of HSS interventions on at least one HSs building block, whereas in Adam et al. (2012) SR, at least 50\% of the evaluations assessed impact of HSS interventions on one HSs building block only.

Focus of SR by Bucagu et al. (2012) was limited to effectiveness of HSS intervention on maternal and newborn health services in Rwanda while focus of this SR is effectiveness of HSS interventions in Africa. Bucagu et al. (2012) did not mention in their study that they assessed their included studies and reports for scientific quality, whereas it is explicit in this SR that critical quality appraisal of all included evaluations was done. While authors of this SR searched minimum acceptable number of databases (Medline and Embase) for relevant literatures, Bucagu et al. (2012) did not do comprehensive search for peer-reviewed articles for their SR because they limited their search for published articles to Medline only.

\section{Discussion and conclusions}

\subsection{Implications of this SR for public health practice}

This SR is not exhaustive in its search for grey literatures. Nevertheless, implication of findings from this SR shall be explored for practice of public health and HSS initiatives. The evaluations that culminated into 11 reports included in this SR appear to lack thorough planning, and consequently could not elaborately capture all data that would have been needed to demonstrate effectiveness or otherwise of HSS interventions. Since GAVI could allocate USD 50,000 for support of HSS proposal development, it should have, from onset endeavoured to allocate $5 \%$ to $10 \%$ of approved grant/funding for study to monitor and evaluate impact of the HSS interventions. This position is buttressed by works of Youngleson et al. (2010), Kebede et al. (2012), and Webster et al. (2012) who through conception, design, and execution of their studies and evaluation of interventions demonstrated effectiveness or otherwise of HSS interventions within a restricted area/population.

The three least targeted and covered HSs building blocks are: leadership and governance (four evaluations); health financing (two evaluations); and health information (three evaluations). Among the three, according to WHO (2010a), leadership and governance, and health information have system-wide effect as they provide basis for complete policy and regulation of the remaining four HSs building blocks. Based on intervention gap in leadership and governance, and health information as revealed by this SR, and importance of their system-wide effects on other HSs building blocks, it will be beneficial if global health funders as well as national governments begin to focus more on strengthening the two. Strengthening of health financing as a building block directly 
or subtly leads to social and financial risk protection for the populace, especially the extremely poor. And in a way, it will contribute to alleviating poverty among the extremely poor.

HS like any other systems is made up of components (building blocks) that are interwoven as shown by WHO (2007, 2010a), and in modified form in this SR as Figure 1. Therefore, holistic approach should be adopted in strengthening HSs in order to achieve synergistic national health outcomes, unlike the way it has been done in the evaluations systematically reviewed for this study. Supporting this position are findings from studies by Duber et al. (2010) and Cohen et al. (2013): They evaluated disease-specific (HIV/AIDS) HSS interventions by PEPFAR funding. The two studies showed that PEPFAR funding as huge as it is, resulted in little or no improvement in non-HIV/AIDS specific national health outcomes in PEPFAR countries.

\subsection{Implications of this SR for public health research}

There is dearth of studies evaluating HSS interventions in Africa as shown by only six quantitative and one qualitative peer-reviewed studies that met inclusion criteria for this SR. There is need for more researches on HSS interventions in Africa as a whole, and specifically in West Africa, since there was no published study from West Africa that was found and included in this SR. One of the weaknesses of this SR is that it inadvertently excluded studies from North Africa; therefore, further SRs should endeavour to include criteria that will incorporate studies from North Africa. Most of the six quantitative studies included in the SR are internally invalid because they did not minimise sources of bias by adjusting for potential confounders. Future researchers into the subject area should endeavour to minimise sources of bias by adjusting for potential confounders where applicable.

This SR did not exhaust search for grey literatures. Further researches should search for grey literatures from website of organisations that are involved in evaluations global health interventions/initiatives.

Most grey literatures included in this SR are not of high quality as shown in Appendix 2. Therefore, future evaluation studies should be designed, conducted and reported in such a way that it will be of good quality when assessed using NICE (2012) quality appraisal for qualitative studies tool or any similar tool.

\subsection{Limitations of this $S R$}

This SR strives to minimise any bias or weakness that may affect its conduct and outcome. Nevertheless, some weaknesses are unavoidably present. Two databases (Medline and Embase) were exhaustively searched for this review. However, there was no exhaustive search for grey literatures, and no expert was consulted for opinion on the subject. In addition, all searches were electronically conducted; thereby missing out any potentially relevant papers/reports that were not available online. All these constitute limitations to this SR and its outcome.

There is weakness introduced into this SR by ten reports (Lewis and Leigh, 2009; Carlson and Karibwami, 2009; Martinez and Karasi, 2009; Alebachew and Ortendahl, 2009a, 2009b; Lewis and Kamanga, 2009; Lewis, 2009a, 2009b; England, 2009; Carlson et al., 2009) produced by HLSP Institute in the UK for GAVI. Since the studies were 
commissioned by GAVI, this most probably explains the reason why the reports are overtly positive in their tone with respect to effectiveness of GAVI HSS interventions. This has potential of misleading and influencing this SR towards concluding that such HSS interventions yielded expected result.

Finally, this SR fails to seek out missing information on methodology (evaluation framework, key questions, study components, guidelines for data collection, sampling method), outcome and/or intervention data from authors of 18 studies/reports. However, the authors, twice (24/03/2014 and 13/04/2014) through e-mail sought out missing methodological information from HLSP Institute on its ten reports for GAVI that are included in this review. The sought-out methodological data is not made available because there was no response from HLSP Institute. This unavailability of methodological data may have caused methodical hole in the analysis, and poor performance of the ten reports in quality appraisal of the HSS intervention reports as shown in summary of critical assessment of included qualitative evaluations. Supposing that the methodological data is available, risk of bias domains with 'unclear risk/not sure' would have been clarified.

Since this SR unintentionally excluded North African countries, therefore, findings from the included studies and reports may not be externally valid across North Africa. Though, most of the reports included in this review have shortcomings, however, their findings may be externally valid within the various African regions where they were carried out.

\subsection{Overall conclusions}

Notwithstanding deficiencies of evaluations included in this SR, findings from the SR have answered the study question: In Africa, to what extent have HSS interventions resulted in improved HSs as measured by indicators of HSs building blocks and distal indicators of HSS? HSS interventions in Africa have not holistically strengthened the HSs building blocks. This explains the reason there is no intervention that simultaneously targeted all six HSs building blocks, just as there is no evaluation that reported holistic improvement in all the four HSS overall outcomes. That is, HSS interventions in Africa have been adopting piece-meal approach to strengthening weak HSs on the continent. Findings from this SR corroborate the position of Adams et al. (2012) that there is need for holistic approach to HSS as well as to assessment of system-wide impact of HSS interventions, for mitigation of any negative effects and behaviour, and for amplification of any possible synergies (de Savigny and Adam, 2009).

It is found out from this SR that HSS interventions at small unit level (district/local government/province/state) easily demonstrated positive impact of such interventions on both intermediate and overall outcomes of HSS. However, such impacts are not obviously demonstrated at national level because they show little or no improvement in overall outcomes of HSS, and national health indicators. From the evaluations reviewed for this SR, it comes to fore that system-level HSS interventions take longer time to demonstrate improvement in overall outcomes of HSS and national health indicators, probably due to weak health information system.

Owing to intervention gap in leadership and governance, and health information building blocks as revealed by this SR, and importance of their system-wide effects on other HS building blocks, it will be beneficial if global health funders as well as national governments begin to focus more on strengthening the duo. 


\subsection{Recommendations}

Based on findings distilled from this SR, the following recommendations are made to advance research and practice of public health, and HSS policy development and implementation:

1 In addition to findings from works of Duber et al. (2010), Pfeiffer et al. (2010), Adam et al. (2012), and Cohen et al. (2013), this SR found out that there was no HSS intervention that concurrently targeted all the six HS building blocks, and none improved all the four overall outcomes of HSS. It is therefore recommended that future HSS interventions should be holistic by simultaneously strengthening all six HSS building blocks and assess impacts on all the four overall outcomes of HSS, even if it means pooling of resources from different sources for the HSS intervention.

2 In situations where it is impossible to adopt holistic approach to HSS interventions, based on intervention gap in leadership and governance, and health information as revealed by this SR, and their system-wide effects on other HSs building blocks (WHO, 2010a), it is therefore recommended that global health funders as well as national governments should always include strengthening of the two HSs building blocks in any of their HSS interventions.

3 It is shown in this SR that there is paucity of researches on HSS in Africa as a whole, specifically in West Africa, because there was no peer-reviewed study from West Africa found and included in this review. It is recommended that studies should be conducted on any HSS interventions in the region to demonstrate effectiveness or otherwise of such interventions.

4 Youngleson et al. (2010), Kebede et al. (2012), and Webster et al. (2012) through conception, design, and conduct of their studies, and evaluation of interventions, demonstrated effectiveness of HSS interventions, whereas the studies that culminated into 11 reports included in this SR appeared not to have been thoroughly planned, and consequently could not elaborately capture all data that were needed to explicitly demonstrate effectiveness or otherwise of such HSS interventions. Therefore, it is recommended that global health funders as well as national governments should always endeavour, from onset, to allocate $5 \%$ to $10 \%$ of approved grant/funding for study to monitor and evaluate impact of the HSS interventions, and capture any emerging lessons from such interventions.

\section{References}

Adam, T., Hsu, J., de Savigny, D., Lavis, J.N., Rottingen, J.A. and Bennett, S. (2012) 'Evaluating health systems strengthening interventions in low-income and middle-income countries: are we asking the right questions?', Health Policy and Planning, Vol. 27, No. 4, pp.9-19.

Alebachew, A. and Ortendahl, C. (2009a) GAVI Health System Strengthening Support Evaluation Study: Ethiopia Case Study, A Report prepared and submitted by HLSP to GAVI, HLSP, 5-23 Old Street, London, EC1V 9HL, UK.

Alebachew, A. and Ortendahl, C. (2009b) GAVI Health System Strengthening Support Evaluation Study: Kenya Desk-based Case Study, A Report prepared and submitted by HLSP to GAVI, HLSP, 5-23 Old Street, London, EC1V 9HL, UK. 
Bucagu, M., Kagubare, J., Basinga, P., Ngabo, F., Timmons, B. and Lee, A. (2012) 'Impact of health systems strengthening on coverage of maternal health services in Rwanda, 2000-2010: a systematic review', Reproductive Health Matters, Vol. 20, No. 39, pp.50-61.

Carlson, C. and Karibwami, A. (2009) GAVI Health System Strengthening Support Evaluation Study: Burundi Case Study, A Report prepared and submitted by HLSP to GAVI, HLSP, 5-23 Old Street, London, EC1V 9HL, UK.

Carlson, C., Maw, H. and Mafuta, E. (2009) GAVI Health System Strengthening Support Evaluation Study: Democratic Republic of Congo Case Study, A Report prepared and submitted by HLSP to GAVI, HLSP, 5-23 Old Street, London, EC1V 9HL, UK.

Cohen, R., Li, Y., Giese, R. and Mancuso, J. (2013) 'An evaluation of the president's emergency plan for aids relief effect on health systems strengthening in Sub-Saharan Africa', Journal Acquired Immune Deficiency Syndrome, Vol. 62, No. 4, pp.471-479.

de Savigny, D. and Adam, T. (2009) Systems Thinking for Health Systems Strengthening, Alliance for Health Policy and Systems Research, World Health Organization, Geneva.

Duber, H., Coates, T., Szekeras, G., Kaji, A. and Lewis, R. (2010) 'Is there an association between PEPFAR funding and improvement in national health indicators in Africa? A retrospective study', Journal of the International AIDS Society, Vol. 13, No. 21, pp.1-9.

England, R. (2009) GAVI Health System Strengthening Support Evaluation Study: Ghana Desk-based Case Study, A Report prepared and submitted by HLSP to GAVI, HLSP, 5-23 Old Street, London, EC1V 9HL, UK.

Hafner, T. and Shiffman, J. (2013) 'The emergence of global attention to health systems strengthening', Health Policy Plan, Vol. 28, No. 1, pp.41-50.

Islam, M. (2007) Health Systems Assessment Approach: A How-To Manual, Submitted to the US Agency for International Development, in collaboration with Health Systems 20/20, Partners for Health Reformplus, Quality Assurance Project, \& Rational Pharmaceutical Management Plus.

Kalk, A., Groos, N., Karasi, J. and Girrbach, E. (2010) 'Health systems strengthening through insurance subsidies: the GFATM experience in Rwanda', Tropical Medicine and International Health, Vol. 15, No. 1, pp.94-97.

Kebede, S., Mantopoulos, J., Ramanadhan, S., Cherlin, E., Gebeyehu, M., Lawson, R. and Bradley, E. (2012) 'Educating leaders in hospital management: a pre-post study in Ethiopian hospitals', Global Public Health: An International Journal for Research, Policy and Practice, Vol. 7, No. 2, pp.164-174.

Lewis, D. (2009a) GAVI Health System Strengthening Support Evaluation Study: Nigeria Desk-based Case Study, A Report prepared and submitted by HLSP to GAVI, HLSP, 5-23 Old Street, London, EC1V 9HL, UK.

Lewis, D. (2009b) GAVI Health System Strengthening Support Evaluation Study: Sierra Leone Desk Study, A Report prepared and submitted by HLSP to GAVI, HLSP, 5-23 Old Street, London, EC1V 9HL, UK.

Lewis, D. and Kamanga, K. (2009) GAVI Health System Strengthening Support Evaluation Study: Zambia Case Study, A Report prepared and submitted by HLSP to GAVI, HLSP, 5-23 Old Street, London, EC1V 9HL, UK.

Lewis, D. and Leigh, B. (2009) GAVI Health System Strengthening Support Evaluation Study: Liberia Case Study, A Report prepared and submitted by HLSP to GAVI, HLSP, 5-23 Old Street, London, EC1V 9HL, UK.

Martinez, J. and Karasi, L. (2009) GAVI Health System Strengthening Support Evaluation Study: Rwanda Case Study, A Report prepared and submitted by HLSP to GAVI, HLSP, 5-23 Old Street, London, EC1V 9HL, UK. 
National Institute for Health and Clinical Excellence (NICE) (2012) Methods for the Development of NICE Public Health Guidance: Process and Methods Guides, 3rd ed., MidCity Place, 71 High Holborn, WC1V 6NA, London [online]

http://www.nice.org.uk/aboutnice/howwework/developingnicepublichealthguidance/ publichealthguidanceprocessandmethodguides/public_health_guidance_process_and_method_ guides.jsp (accessed 10 February 2014).

Pfeiffer, J., Montoya, P., Baptista, A., Karagianis, M., Pugas, M., Micek, M., Johnson, W., Sherr, K., Gimbel, S., Baird, S., Lambdin, B. and Gloyd, S. (2010) 'Integration of HIV/AIDS services into African primary health care: lessons learned for health system strengthening in Mozambique - a case study', Journal of the International AIDS Society, Vol. 13, No. 3, pp.1-9.

Richards, T. (2004) 'Poor countries lack relevant health information, says Cochrane editor', $B M J$, Vol. 328, No. 7435, p.310.

Sekhri, N. (2006) From Funding to Action: Strengthening Healthcare Systems in Sub-Saharan Africa, World Economic Forum White Paper Centre for Public-Private Partnership Global Health Initiative [online] http://www.bd.com/globalhealth/initiative/pdfs/whitepaper.pdf (accessed 24 February 2014).

Shakarishvili, G., Lansang, M., Mitta, V., Bornemisza, O., Blakely, M. and Kley, N. (2011) 'Health systems strengthening: a common classification and framework for investment analysis', Health Policy Plan, Vol. 26, No. 4, pp.316-326, pp.316-326.

Smith, O., Gbangbade, S., Hounsa, A. and Miller-Franco, L. (2005) Benin: System-wide Effects of the Global Fund: Interim Findings, The Partners for Health Reform plus Project, Abt Associates Inc., Bethesda, MD.

Tanahashi, T. (1978) 'Health services coverage and its evaluation', Bulletin of the World Health Organization, Vol. 56, No. 2, pp.295-303.

Webster, P., Sibanyoni, M., Malekutu, D., Mate, K., Venter, F., Barker, P. and Moleko, W. (2012) 'Using quality improvement to accelerate highly active antiretroviral treatment coverage in South Africa', BMJ Qual. Saf., Vol. 21, No. 1, pp.315-324.

World Bank (2004) World Development Report 2004: Making Services Work for Poor People, World Bank and Oxford University Press, Washington DC.

World Health Organization (WHO) (2000) The World Health Report 2000: Health Systems Improving Performance, WHO Press, 20 Avenue Appia, 1211 Geneva 27, Switzerland.

World Health Organization (WHO) (2006) World Health Report 2006: Working Together for Health, WHO Press, 20 Avenue Appia, 1211 Geneva 27, Switzerland.

World Health Organization (WHO) (2007) Everybody's Business: Strengthening Health Systems to Improve Health Outcomes: WHO's Framework for Action, WHO Press, 20 Avenue Appia, 1211 Geneva 27, Switzerland.

World Health Organization (WHO) (2008) The World Health Report 2008. Primary Health Care Now More Than Ever, WHO Press, 20 Avenue Appia, 1211 Geneva 27, Switzerland.

World Health Organization (WHO) (2010a) Monitoring the Building Blocks of Health Systems: A Handbook of Indicators and their Measurement Strategies, WHO Press, 20 Avenue Appia, 1211 Geneva 27, Switzerland.

World Health Organization (WHO) (2010b) Health System Strengthening - Current Trends and Challenges, Executive Board 128th session, Geneva, December 2010. (EB128/37) [online] http://apps.who.int/gb/ebwha/pdf_files/EB128/B128_37en.pdf (accessed 20 February 2014).

Youngleson, M.S., Nkurunziza, P., Jennings, K., Arendse, J., Mate, K.S. et al. (2010) 'Improving a mother to child HIV transmission programme through health system redesign: quality improvement, protocol adjustment and resource addition', PLoS ONE, Vol. 5, No. 11, p.e13891, DOI: 10.1371/journal.pone.0013891. 
66

O.O. Fatodu and O.O. Oyenuga

\section{Appendix 1}

Table A1 Extracted data and results from studies and reports included in the review

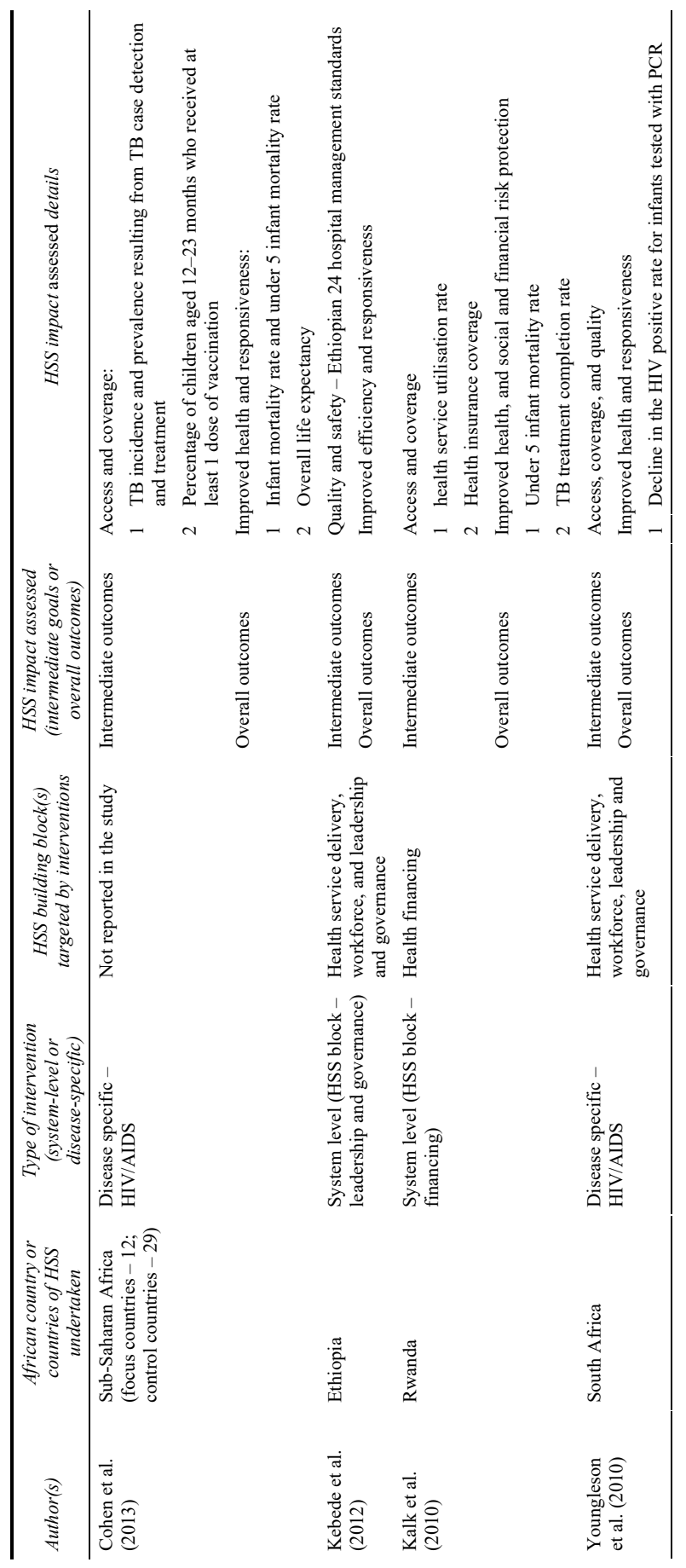


Table A1 Extracted data and results from studies and reports included in the review (continued)

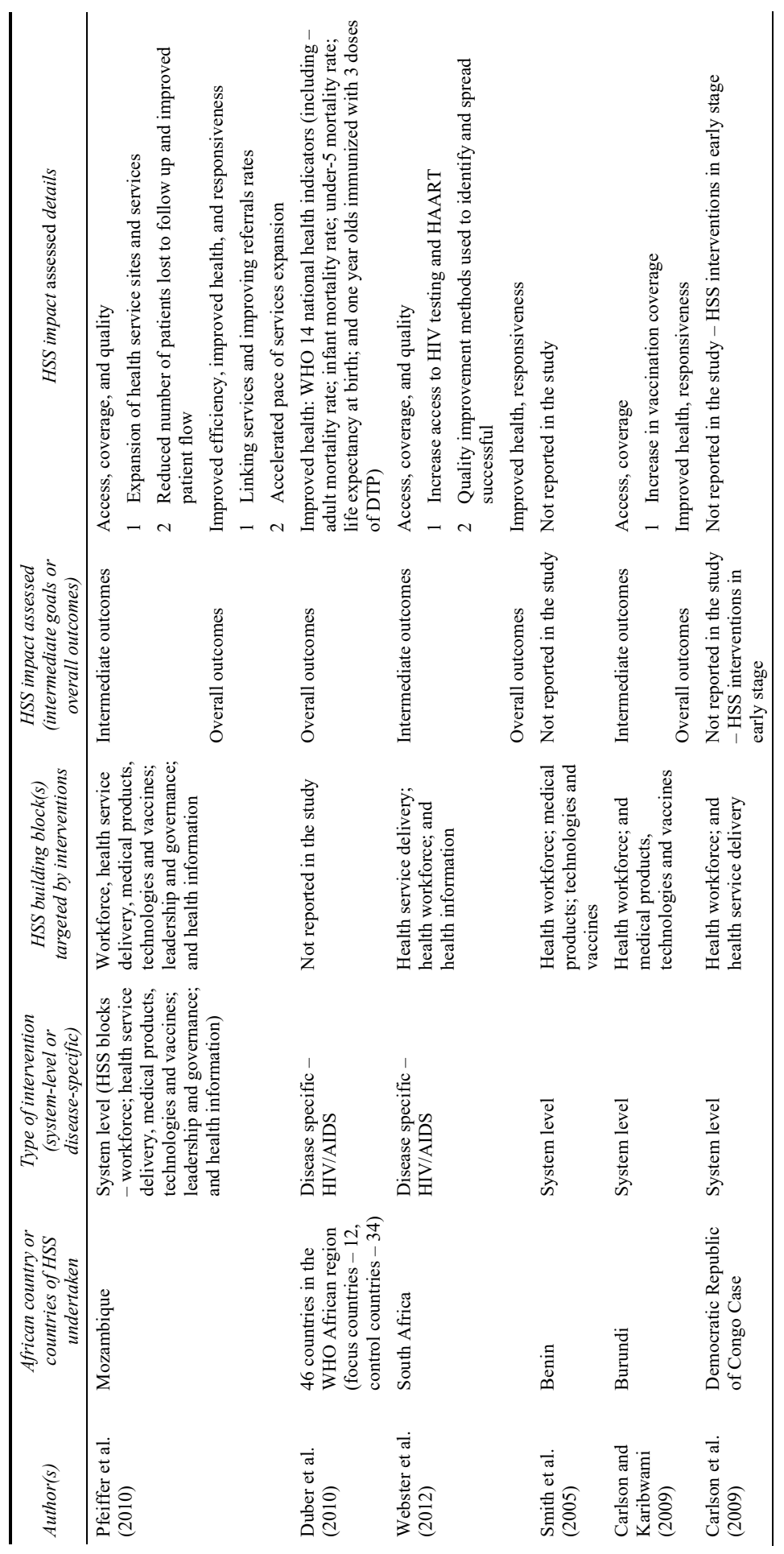


Table A1 Extracted data and results from studies and reports included in the review (continued)

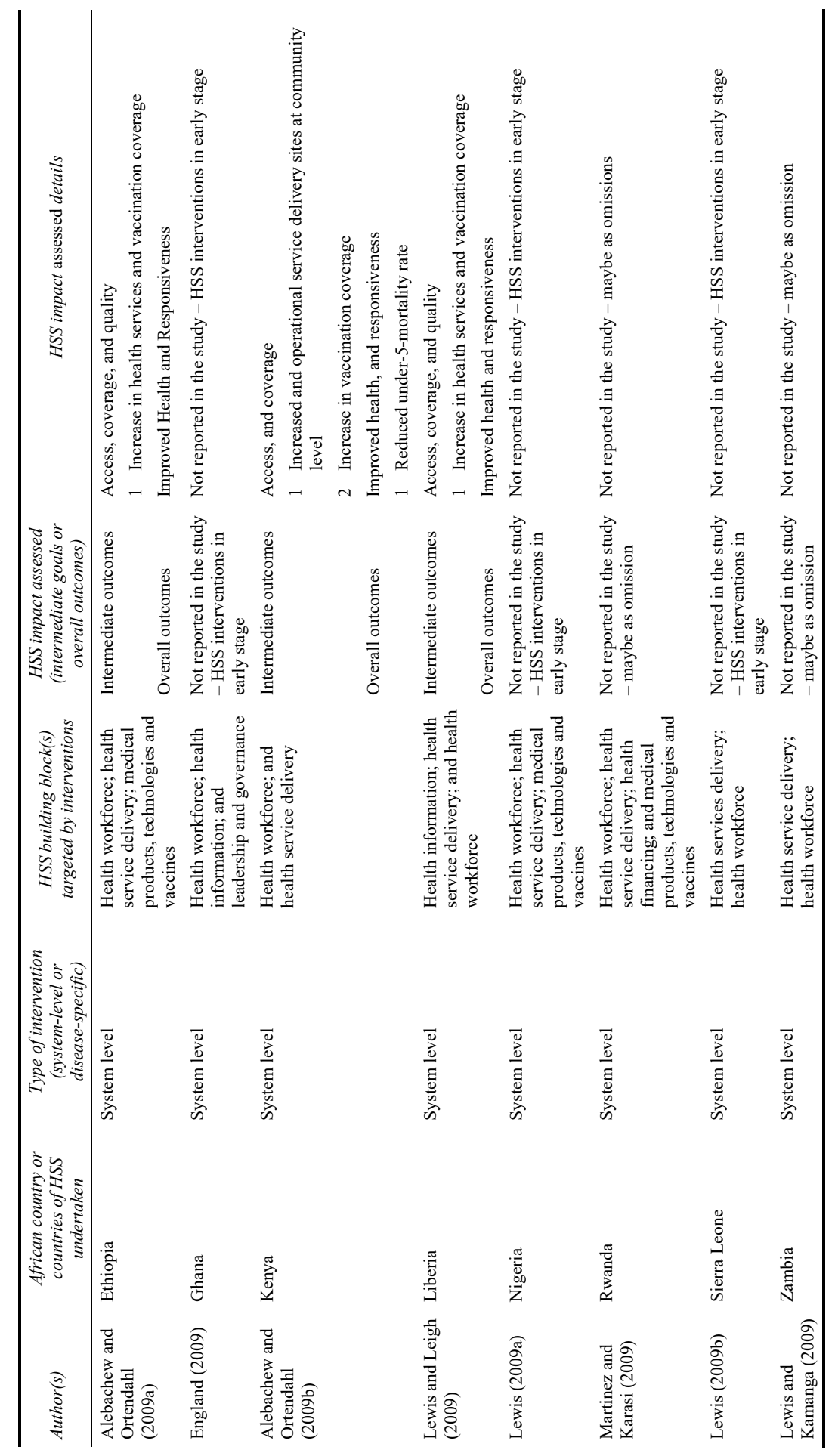




\section{Appendix 2}

Table A2 Summary of critical assessment of included qualitative study and reports

\begin{tabular}{|c|c|c|c|c|c|}
\hline Author(s) & Awarded points & Grade & Author(s) & Awarded points & Grade \\
\hline $\begin{array}{l}\text { Lewis and Leigh } \\
\text { (2009) }\end{array}$ & 9 & ++ & $\begin{array}{c}\text { Kalk et al. } \\
(2010)\end{array}$ & 3 & - \\
\hline $\begin{array}{l}\text { Carlson and } \\
\text { Karibwami } \\
(2009)\end{array}$ & 8 & + & Lewis (2009a) & 4 & - \\
\hline $\begin{array}{l}\text { Martinez and } \\
\text { Karasi (2009) }\end{array}$ & 6 & + & England (2009) & 4 & - \\
\hline $\begin{array}{l}\text { Alebachew and } \\
\text { Ortendahl } \\
(2009 a)\end{array}$ & 7 & + & Lewis (2009b) & 2 & - \\
\hline $\begin{array}{l}\text { Lewis and } \\
\text { Kamanga (2009) }\end{array}$ & 8 & + & $\begin{array}{l}\text { Smith et al. } \\
(2005)\end{array}$ & 11 & ++ \\
\hline $\begin{array}{l}\text { Alebachew and } \\
\text { Ortendahl } \\
(2009 b)\end{array}$ & 3 & - & $\begin{array}{l}\text { Carlson et al. } \\
\quad(2009)\end{array}$ & 9 & ++ \\
\hline
\end{tabular}

Notes: $9-15=(++)=$ All or most of the checklist criteria have been fulfilled; where they have not been fulfilled the conclusions are very unlikely to alter. $6-8=(+)=$ Some of the checklist criteria have been fulfilled, where they have not been fulfilled, or not adequately described, the conclusions are unlikely to alter. $1-5=(-)=$ Few or no checklist criteria have been fulfilled and the conclusions are likely or very likely to alter.

Source: Adapted legend from NICE (2012)

\section{Appendix 3}

Table A3 Summary of critical assessment of included quantitative evaluations

\begin{tabular}{lcc}
\hline Authors & Awarded points & Grade \\
\hline Cohen et al. (2013) & 23 & + \\
Kebede et al. (2012) & 29 & ++ \\
Webster et al. (2012) & 27 & ++ \\
Youngleson et al. (2010) & 23 & + \\
Pfeiffer et al. (2010) & 18 & + \\
Duber et al. (2010) & 26 & ++ \\
\hline
\end{tabular}

Notes: $26-40=(++)=$ All or most of the checklist criteria have been fulfilled; where they have not been fulfilled the conclusions are very unlikely to alter.

$16-25=(+)=$ Some of the checklist criteria have been fulfilled, where they have not been fulfilled, or not adequately described, the conclusions are unlikely to alter.

Source: Adapted legend from NICE (2012) 